\section{Worldwide ageing programme needed}

Sir - I read with interest the News story about the issues raised by Japan's demography, showing that a dramatic increase in population age is being accompanied by a decrease in the number of financially productive individuals (Nature 385, 379; 1997).

The extrapolations that can be made from Japan to other countries and possibly to the entire world are striking. Scientists are actively searching for ways to increase the human lifespan and at the same time to solve the medical problems of old age, such as degenerative disease and dementia. But old people must also have a socially and economically acceptable quality of life. Several questions need answering. Is it reasonable that scientists should try to increase the length of human life without a parallel change in the mechanisms by which society treats the aged? Would it be ethical to cease these efforts? Can state health insurance systems insure everybody against the as yet inevitable diseases associated with old age, without violating the very principle of 'insurance'? Can an old person still be active and productive for society?

The answers to these questions cannot come from single disciplines. Some international organization should take responsibility for coordinating all aspects of ageing research, cultural, social and biological. Unesco has shown itself effective and successful in a great number of worldwide projects carried out by its three major sectors - education, science and culture. Could Unesco unite efforts in a global project of providing, for the year 2000 , a convincing model to tackle the population ageing problem? Or do we rather need a new organization, a United Nations Programme for Aged Persons, perhaps?

\section{Angelo Azzi}

Department of Biochemistry,

University of Bern,

Bern, Switzerland

e-mail:azzi@mci.unibe.ch

\section{Data available}

Sir-Our laboratories are currently determining the complete nucleotide sequences of the genomes of the obligate human pathogens Streptococcus pyogenes and Neisseria gonorrhoeae, sponsored by the National Institute of Allergy and Infectious Diseases (NIAID) of the US National Institutes of Health $(\mathrm{NIH})$. As we have been committed from the beginning of these projects to an early data-release policy for the benefit of the scientific community, we wish to notify the community of the availability of preliminary nucleotide sequence databases for these continuing genome sequencing projects. Contrary to recent assertions in Science $(275,777 ; 7$ February 1997), these data have been freely available on the Internet since mid-September 1996 and may be obtained at http://www.genome.ou.edu.

The data collected for both organisms have been compiled into Blast databases that are searchable from our Web site; alternatively, the data can be downloaded via FTP from this site. These databases represent raw, assembled data that are not currently proof-read, provided in a series of contiguous segments (contigs), which will decrease in number as the gaps between the contigs close. All data should be considered preliminary until gaps are closed and proofreading and annotation are complete.

Investigators using any of this information are asked to acknowledge these microbial pathogen genome projects, sponsored by NIAID/NIH. Further information can be obtained from J.J.F. ( $S$. pyogenes), or D.W.D. (N. gonorrhoeae) or from B.A.R. (both organisms) at the addresses below.

\section{Joseph J. Ferretti}

\section{David W. Dyer}

Department of Microbiology and Immunology, Oklahoma University Health Sciences Center, Oklahoma City, Oklahoma 73104, USA

\section{Bruce A. Roe}

Department of Chemistry and Biochemistry, University of Oklahoma,

Norman, Oklahoma 73019, USA

\section{Worthless ranking}

Sir - Steve Jones's analogy between Gogol's The Government Inspector and the Research Assessment Exercise (Nature 385, $311 ; 1997)$ was just beautiful. Perhaps the point is that science is done by individuals, not by departments. To rank science by department is about as clever as ranking journals by their impact factor; neither procedure tells one anything very interesting (except to the bureaucratic mind). The former is a crude average over a group of very different people which tells one little about the science done by each of them. The latter is a method of ranking the commercial success of journal editors, not a method for assessing the worth of individual scientists. (My own department was 5-star, so I have no axe to grind.)

\section{David Colquhoun}

Department of Pharmacology,

University College London,

Gower Street,

London WC1E 6BT, UK
Marijuana as a medicine

Sir - Your recent News story on marijuana (Nature 385, 756; 1997) was factual and objective but, as in most of the other media coverage of the subject of smoked cannabis versus oral $\Delta$-9-tetrahydrocannabinol (THC) (Marinol), an important point has been omitted.

The cannabis plant is a complex mixture of substances that include at least 60 different cannabinoids, some of which have been shown to have pharmacological activities of their own. For example, one such component, cannabidiol (CBD) can affect the metabolism of THC and, therefore, in some individuals may improve the anti-emetic properties of the latter. Interestingly, cannabidiol is usually more abundant in most samples of marijuana.

Given such facts, it seems rather unenlightened to dismiss the reports from cancer and AIDS patients who claim greater benefit from smoked marijuana than from oral THC as simply a ploy to facilitate government approval for medical use. Sumner Burstein

Department of Biochemistry and Molecular Biology, University of Massachusetts Medical School, Worcester, Massachusetts 01655, USA

\section{Hantavirus in Argentina}

Sir - You say in a recent News article about staff and budget cuts in Argentinian health laboratories that there are 20 new cases of hantavirus infection each week (Nature 385, 376; 1997).

We are working at a private hospital in the city of Bariloche, where most of the cases have been treated. Up to 15 December 1996, 77 cases of HPS (hantavirus pulmonary syndrome) had been reported and confirmed in Argentina. Of these, 37 people died and the rest recovered (D. Enria et al. Medicina 56, 709-711; 1996). During 1996 , a total of 22 cases were notified in southern Argentina. But no new cases have been reported this year.

The economy of the whole lake district on the eastern slopes of the southern Andes and centred on Bariloche is based on tourism. The region has suffered its worst summer season ever because of misleading media accounts about the extent and severity of the hantavirus outbreak.

Nevertheless, we are deeply concerned about the staff and budget cuts to the National Institute for Microbiology, an institution that has played an important role in handling the hantavirus outbreak. Carlos M. Barclay, Gabriela Rubinstein Sanatorio San Carlos, Av. E. Bustillo Km.1, 8400 Bariloche, Argentina 